\title{
新型两亲性嵌段共聚物导向合成有序大孔径介孔材料的研究进展
}

$$
\text { 罗 维 }{ }^{1,2} \text {, 魏 晶 }{ }^{3} \text {, 邓勇辉 }{ }^{3} \text {, 李宇慧 }{ }^{3} \text {, 王连军 }{ }^{1} \text {, 赵 涛 }{ }^{1} \text {, 江 莞 }{ }^{1}
$$

(1. 东华大学 纤维材料改性国家重点实验室, 材料科学与工程学院, 上海 201620; 2. 中国科学院 上海硅酸盐研究 所, 上海 200050; 3.复旦大学 化学系, 上海 200433)

摘 要: 自从 1992 年首次报道介孔氧化硅分子篮 M41S 系列以来, 人们采用各种商业化表面活性剂为模板, 合成了 多种骨架组成、丰富的有序介观结构、不同孔径尺寸的介孔材料，并将其应用在能源、环境、催化等诸多领域。然 而, 由于常规商业化模板剂的分子量大小有限, 合成的介孔材料具有较小的孔径 $(<8.0 \mathrm{~nm})$, 从而极大地限制了其 面对大尺寸客体分子的相关应用。此外，利用常规模板剂难以合成出具有晶化墙壁的介孔金属氧化物材料。近年来， 大分子量两亲性嵌段共聚物相继被报道用来合成新型介孔材料，本文将综述基于这种嵌段共聚物为模板剂合成各 种具有大孔径和晶化墙壁介孔材料的研究进展。

关 键 词: 介孔材料; 嵌段共聚物; 软模板; 自组装; 综述

中图分类号: TQ174 文献标识码: A

\section{Progress on the Fabrication of Ordered Mesoporous Materials with Large Pores by Using Novel Amphiphilic Block Copolymers as Templates}

\author{
LUO Wei ${ }^{1,2}$, WEI Jing ${ }^{3}$, DENG Yong-Hui ${ }^{3}$, LI Yu-Hui ${ }^{3}$, WANG Lian-Jun ${ }^{1}$, ZHAO Tao ${ }^{1}$, JIANG Wan ${ }^{1}$ \\ (1. State Key Laboratory for Modification of Chemical Fibers and Polymer Materials, College of Materials Science and Engi- \\ neering, Donghua University, Shanghai 201620, China; 2. Shanghai Institute of Ceramics, Chinese Academy of Sciences, \\ Shanghai 200050, China; 3. Department of Chemistry, Fudan University, Shanghai 200433, China)
}

\begin{abstract}
Ever since mesoporous silica M41S were reported in 1992, various mesoporous materials with diverse frameworks, abundant ordered mesostructures and different pore size have been synthesized by using commercial surfactants as template, due to their great potential for applications in energy storage and conversion, environmental remedy, catalysis. However, these mesoporous materials usually have pore sizes of less than $8.0 \mathrm{~nm}$ resulting from the low molecule weight of commercial templates, which greatly limit their applications in large guest objects. Moreover, these commercial templates are hard to synthesize ordered mesoporous metal oxides with high crystallinity. Recently, various amphiphilic copolymers with high molecule weight were reported to fabricate novel mesoporous materials, the recent research advances about their templated ordered mesoporous materials with large pore size and highly crystallized framework was reviewd in this paper.
\end{abstract}

Key words: mesoporous material; block copolymer; soft template; self-assembly; review

收稿日期: 2016-05-10; 收到修改稿日期：2016-06-14

基金项目: 国家自然科学基金(51402049, 51432004); 上海市科委项目(14ZR1400600); 高等学校学科创新引智计划(111-2-04); 中 央高校基本科研业务费(2232015D3-06)

National Natural Science Foundation of China (51402049, 51432004); Shanghai Committee of Science and Technology, China (14ZR1400600); Programme of Introducing Talents of Discipline to Universities (111-2-04); Fundamental Research Funds for the Central Universities (2232015D3-06)

作者简介: 罗 维(1983-), 男，博士，讲师. E-mail: wluo@dhu.edu.cn

通讯作者: 江 莞, 教授.E-mail: wanjiang@dhu.edu.cn 
有序介孔材料(ordered mesoporous material)是 一类孔径介于 $2 \sim 50 \mathrm{~nm}$ 之间, 且孔道结构排列有 序的多孔材料 ${ }^{[1]}$ 。这类材料具有较大且均一的孔径 尺寸, 多元化骨架结构以及较高比表面积 ${ }^{[2-4]}$ 等特 点, 在新能源 ${ }^{[5-9]}$ 、催化 ${ }^{[10-13]}$ 、吸附与分离 ${ }^{[14-17]}$ 、传 感 ${ }^{[18-19]}$ 和药物释放 ${ }^{[20-22]}$ 等领域应用很广。自从 1992 年 Mobil 公司的研究人员选用烷基季铵盐阳离子表 面活性剂作为模板剂, 引导无机硅物种自组装, 首 次合成了介孔氧化硅分子篮 M41S 系列以来, 很多 科学家采用超分子为模板制备了一系列具有不同骨 架组成、多种有序孔道结构和可调孔径尺寸的介孔 材料。

有序介孔材料的合成方法可以分为两类：硬模 板法和软模板法 ${ }^{[23]}$ 。硬模板法是将所需要制备材料 的前驱物分子灌注到预先合成好的具有介观结构及 刚性骨架模板的孔道中, 再原位转化形成目标产物, 最后通过焙烧或者刻蚀法除去模板剂, 就可以得到 所需要的介孔材料。这种方法一步形成介观相, 适 应面广, 不需要前驱物分子与模板剂之间有相互作 用, 但是合成步骤繁琐, 不适合大批量制备。软模板 法是合成有序介孔材料使用最广泛的方法, 它利用 柔性的表面活性剂分子(即模板剂)与目标产物的前 驱物分子之间较强的作用力(氢键、静电力等), 协同 组装形成有机一无机或有机一有机复合的介观有序稳 定结构, 最后将复合材料中的模板剂通过焙烧或者 萃取等方式除去, 就能够得到孔道排列高度有序的 介孔材料。这种方法相对来说省时省力, 可以通过 调整软模板剂的种类、合成条件以及加入添加剂等 方式来精确控制所合成材料的介观有序结构、孔径 尺寸、比表面积等。随着人们对于软模板法合成介 孔材料的不断研究, 人们对于其形成机理的认识越 来越深, 最终发现介孔材料的孔径与软模板剂的疏 水端体积大小有着很大关系 ${ }^{[24-25]}$, 而孔壁的厚度则 取决于亲水端的大小 ${ }^{[2]}$ 。种类繁多的有序介观结构 则可以通过液晶(LC)理论中的有效堆积参数 $g(g=$ $\left.V_{0} / a_{0} l\right)$ 来半定量地解释, 公式中的 $V_{0}$ 是 $\mathrm{CH}$ 链及链 间助溶剂所占的总体积, $a_{0}$ 为胶团表面极性头所占 据的有效面积, $l$ 为 $\mathrm{CH}$ 链的有效链长, 利用该公式 可以预测并合成一些所需要或特定的介观结构 ${ }^{[26]}$ 。

在介孔材料研究初期, 阳离子型(十六烷基三 甲基溴(氯)化铵、双子座及流星锤型表面活性剂

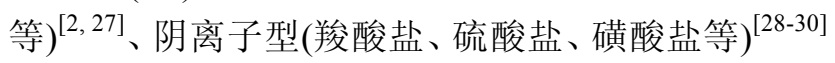
以及非离子型表面活性剂(主要是嵌段共聚物 $)^{[2,31]}$ 都被作为软模板剂来合成了一系列高度有序的介孔 材料。这些模板剂都属于商业化产品, 通常来说分
子量较小, 疏水端的体积也有限, 所以制备出的介 孔材料孔径最大在 $10 \mathrm{~nm}$ 左右, 不能满足一些涉及 到更大分子的应用领域(例如大分子催化、生物分子 吸附等)。虽然可以通过添加有机膨胀剂(如 1, 3, 5三甲基苯)的方式，使疏水的亲油性分子进入表面 活性剂的疏水基团中，从而增加整个疏水基团的体 积, 最终达到扩大孔径的目的。但是这种方法不会 改变介孔材料的晶胞尺寸, 仅仅是通过畸变的方式来 增加孔径, 对材料的介观有序性有较大的影响 ${ }^{[2-33]}$ 。

其次, 这些商业化模板剂的亲水端和疏水端尺寸比 例不能根据实际需要进行自由调控, 只能合成出孔 径与孔壁尺寸比例固定的介孔材料, 也在一定程度 上影响了其应用效果。再者, 这些模板剂的含氧量 较高, 加热易于分解, 当使用这一类模板剂合成介 孔金属氧化物时，在高温晶化或高温焙烧除去模板 剂的过程中，金属氧化物骨架很容易失去模板剂的 支撑而造成有序介观结构妇塌。因此, 使用传统商 业化软模板剂较难合成具有晶化骨架结构的介孔金 属氧化物, 而大多数金属氧化物的优良性能都是建 立在具有高度晶化骨架的基础上的。

为了解决上述问题, 获得孔径更大的介孔材料 和具有晶化骨架的介孔金属氧化物, 有科学家尝试 在实验室设计并制备了大分子量两亲性嵌段共聚物 作为新型软模板剂来合成介孔材料: 通过原子转移 自由基聚合等高分子聚合技术合成模板剂，通过调 节聚合反应的合成参数(单体加入量、反应时间和温 度等)来对嵌段共聚物的组成和分子量进行自由调 控, 改变疏水端和亲水端的体积, 进而在很宽的范 围内对介孔材料的孔径以及墙壁厚度进行调节; 这 类嵌段共聚物经过焙烧后的残碳量很高, 在介孔金 属氧化物墙壁高温晶化的过程中, 嵌段共聚物可以 碳化并转化为碳颗粒, 填充在孔道里发挥支撑作用, 有效地避免有序介观结构的妇塌; 这类疏水端和亲 水端比例可调的嵌段共聚物可以通过调变有效堆积 参数来得到更多种类的有序介观结构, 制备出更加 贴近实际应用的有序介孔材料。因此，设计并合成 新型两亲性大分子量嵌段共聚物, 并以其为模板剂, 制备传统模板剂所不能合成的新型有序介孔材料, 能够丰富介孔材料族群, 进一步探索新型介观结 构。本文综述了以新型两亲性嵌段共聚物为模板剂 制备有序介孔材料的最新进展。

\section{1 大孔径介孔氧化硅材料的合成}

已经商品化的聚醚类嵌段共聚物的溶解性较好, 在水和乙醇等常用溶剂中都能充分溶解, 因此常规 
介孔二氧化硅材料(如 SBA-15 等)都可以在水或乙 醇体系中进行合成。不同的是，实验室设计并制备 的新型两亲性嵌段共聚物通常具有疏水性更强的疏 水端, 以及较高的分子量, 使得这些嵌段共聚物无 法溶解在水、乙醇等常规溶剂中, 所以只能采用其 他种类的有机溶剂(如四氢呋喃等)来进行合成。一 般可以采用溶剂挥发诱导自组装(evaporation induced self assembly, EISA) ${ }^{[34-35]}$ 和溶剂挥发诱导聚集组装 (solvent evaporation induced aggregating assembly, EIAA $)^{[36-38]}$ 的方法来制备新型有序大孔径介孔氧 化硅材料。

EISA 法可以得到膜状或者单片状形貌的材料, 该方法利用溶剂的缓慢挥发, 使得模板剂和前驱体 浓度不断增大, 最终达到临界胶束浓度, 就可以实 现从溶液相到液晶相的转变, 待前驱体骨架进一步 交联使得液晶相固定, 最终得到刚性有序介观结构。 Brinker 等 ${ }^{[39-41]}$ 选用分子量不同的双嵌段共聚物聚环 氧乙烷-block-聚苯乙烯 $\left(\mathrm{PEO}_{109}-b-\mathrm{PS}_{35}\right.$ 和 $\mathrm{PEO}_{100}-b$ $\mathrm{PS}_{230}$ ) 作为模板剂合成了不同孔径大小的介孔氧化 硅材料, 发现随着 PS 链段的增长, 孔径也迅速增加。 但是这种材料的大孔径尺寸孔道是完全孤立的, 具 有闭孔结构, 无法被利用。随后, Deng 等 ${ }^{[42]}$ 通过原子 转移自由基聚合法合成了大分子量的两亲型嵌段共 聚物 $\mathrm{PEO}_{125}-b-\mathrm{PS}_{230}\left(\mathrm{Mw}=29700 \mathrm{~mol}^{-1}\right)$, 并利用其作 为模板剂, 正硅酸乙酯(TEOS)为硅源, 通过 EISA 法 合成出了具有面心立方结构的介孔氧化硅材料, 其 孔径达到 $30.8 \mathrm{~nm}$ ，且孔道均为开放结构(图 1)。

EISA 法一般在液-固(溶液-基底)界面才能够形 成高度有序的介观结构, 所以在制备过程中, 液面 要尽量薄, 才能够得到有序性较好的材料, 这极大 地影响了材料的制备效率, 使其只适于小规模合成 介孔材料。最近, Deng 等 ${ }^{[36]}$ 开发了一种新颖的 EIAA 法来合成三维有序面心立方结构的介孔氧化硅材料

(图 2), 以自合成两亲性嵌段共聚物聚环氧乙烷block-聚甲基丙烯酸甲酯(PEO- $b$-PMMA)为模板剂, TEOS 用作硅前驱体, 四氢呋喃与水为混合溶剂。在 合成过程中，模板剂的良溶剂四氢呋喃不断挥发， 导致混合溶剂对于模板剂的溶解度逐渐下降，从而 在两种溶剂的界面上形成氧化硅寡聚物/PEO- $b$ PMMA 球形复合胶束, 这些胶束再进一步聚集组装 形成具有有序介观结构的氧化硅/嵌段共聚物纳米 复合颗粒。这种方法与 EISA 法有着明显的不同: EISA 法中使用了大量的有机溶剂，只允许存在少 量水; 而 EIAA 法中则有较大量的水存在于初始溶 剂中, 有序介观结构是在水和四氢呋喃的液一液界 面形成的。随后经过水热处理和空气气氛焙烧除去 嵌段共聚物后，可以得到具有大孔径 $(37 \mathrm{~nm})$ 和大窗
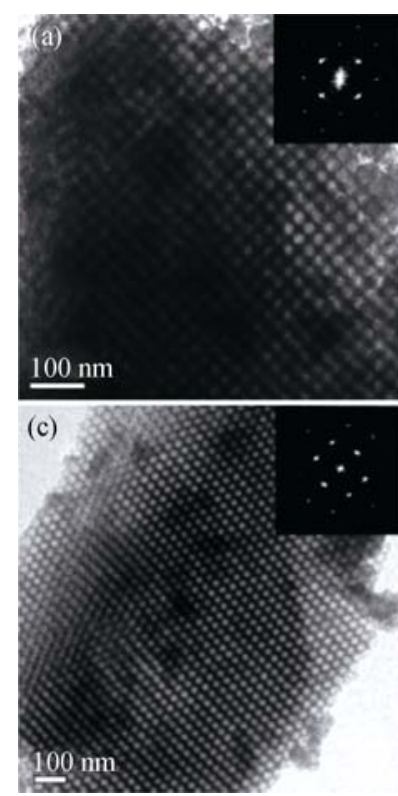

图 1 大孔径有序介孔氧化硅的透射电镜照片 ${ }^{[42]}$

Fig. 1 TEM images of ordered mesoporous silica with large pores $^{[42]}$ (a)

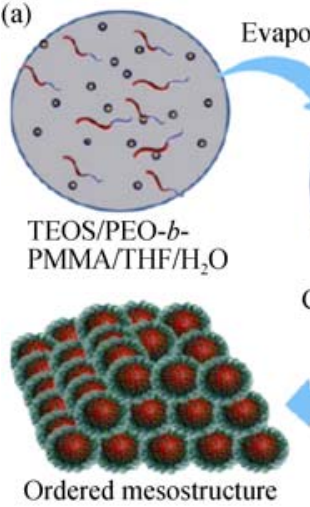

Evaporation of THF

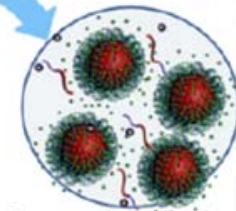

Composite micelles

Aggregation and assembly

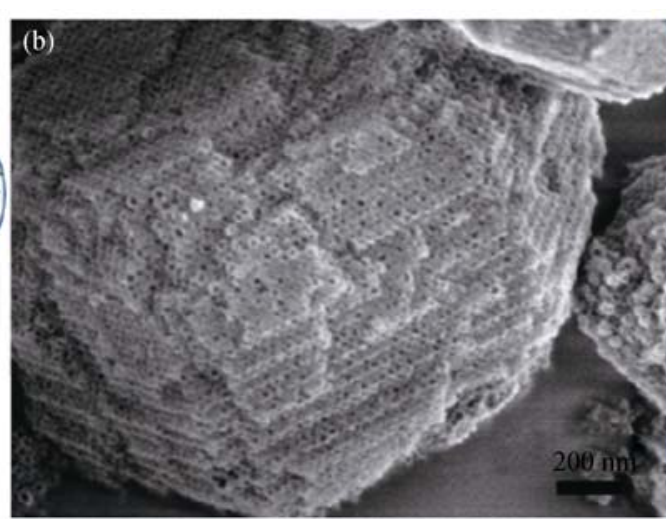

图 2 EIAA 法合成有序介孔氧化硅的机理示意图(a)和扫描电镜照片(b) ${ }^{[36]}$

Fig. 2 Schematic illustration of the formation process (a) and SEM image of mesoporous silica via EIAA approach (b) ${ }^{[36]}$ 
口(8.7 nm)的有序的介孔氧化硅材料(图 3), 比表面 积和孔容分别达到 $508 \mathrm{~m}^{2} / \mathrm{g}$ 和 $1.46 \mathrm{~cm}^{3} / \mathrm{g}$ 。此外, 他 们还在合成体系中加入十六烷基三甲基溴化铵 $(\mathrm{CTAB})$ 作为共模板剂, 得到双介孔结构的氧化硅材 料, 其双介孔结构分别来源于 CTAB 和 PEO- $b$ PMMA 所造的孔, 尺寸分布在 2.5 和 $20 \mathrm{~nm}$, 其中较小 的介孔均匀分布在大介孔氧化硅的墙壁中, 其孔径尺 寸可通过采用不同碳链长短的烷基季铵盐来进行调 节 $^{[37]}$ 。该课题组还尝试在合成体系中施加剪切力, 制备得到均匀的氧化硅纳米管, 管状结构来源于球 形胶束作为构建砌块在剪切力作用下的组装。如果 在体系中再加入少量的有机硅烷(1，2-二(三乙氧基 甲基硅烷)乙烯, BTEE), 就可以控制组装, 形成大 范围有序的二维六方管状阵列, 这种结构包含两种 不同的孔, 即中空氧化硅纳米管内部的圆柱形空腔 和氧化硅纳米管阵列中管与管之间的堆积孔 ${ }^{[38]}$ 。

\section{2 大孔径介孔碳材料的合成}

早期的介孔碳材料一般是利用有序介孔氧化硅 作为硬模板, 通过纳米浇注法来合成的。直到 2004 年, Dai 等 ${ }^{[43]}$ 首次利用软模板法合成了有序介孔碳 材料, 他们选用了大分子量的新型两亲性嵌段共聚 物聚苯乙烯-block-聚(4-乙烯基吡啶)(PS- $b$-P4VP, 总 分子量 $23300 \mathrm{~g} / \mathrm{mol}$ ) 作为模板剂, 间苯二酚/甲醛体 系作为碳源。在合成过程中, 先利用 PS- $b$-P4VP 与 间苯二酚之间的氢键作用, 在基底上自组装形成有 序复合介观结构, 再通过熏蒸甲醛蒸汽, 使其与间 苯二酚原位聚合形成刚性的三维网状结构, 将有序 高分子骨架固定下来。然后经过惰性气氛下高温焙 烧, 嵌段共聚物分解, 碳前驱体碳化形成碳骨架。得 到的介孔碳膜孔径约为 $35 \mathrm{~nm}$ (图 3)。相比于当时得 到的其它介孔碳材料, 孔径有了显著提升, 并且孔
道取向都是垂直于基底的，有利于物质的传输。随 后, Fan 等 ${ }^{[44]}$ 同样选用了 PS- $b$-P4VP 作为模板剂, 松 二糖、棉子糖、葡萄糖等碳水化合物作为碳前驱体, 通过旋涂法实现自组装过程，得到的有机一有机复 合膜材料经过有机溶剂蒸汽处理, 然后在惰性气氛 中焙烧除去模板剂并碳化骨架, 最终得到的碳薄膜 具有无序排列的介孔孔道，孔径大于 $20 \mathrm{~nm}$, 这一 工作显著扩大了碳前驱体的范围。

Deng 等 ${ }^{[42]}$ 也利用实验室自合成的 PEO- $b$-PS 作 为软模板剂, 低阶酚醛树脂为碳前驱体, 并利用其 与模板剂 PEO 端的氢键相互作用互相连接。使用 EISA 法, 通过有机-有机自组装形成 PEO- $b$-PS/酚 醛树脂有序介观结构, 再经过 $100^{\circ} \mathrm{C}$ 热处理将高分 子骨架进一步交联, 然后在惰性气氛下焙烧, 去除模 板剂和碳化酚醛树脂骨架，就可以得到介观结构高度 有序(面心立方密堆积, fcc)且具有大尺寸 $(22.7 \mathrm{~nm}$ )球 形孔道结构的介孔碳材料, 孔道之间的连通性也很 好。随后, 他们还通过调节 PEO- $b$-PS 合成过程中的 聚合时间, 得到了不同分子量的 PEO- $b$-PS, 并作为 模板剂合成了具有不同孔径尺寸 $(12$ 33 nm) 与孔壁 厚度 $(5 \sim 11 \mathrm{~nm})$ 的有序介孔碳材料 ${ }^{[36]}$ 。

提高介孔碳材料的墙壁厚度不仅可以提供更好 的化学和机械稳定性, 而且易于对材料功能化。为 了得到这种材料, Deng 等 ${ }^{[45]}$ 利用 PEO- $b$-PMMA 为 软模板, 低阶酚醛树脂为碳前驱体, 通过 EISA 法成 功得到了具有面心立方介观结构的有序介孔碳材料, 孔径约为 $10.5 \mathrm{~nm}$, 并且该材料的孔壁厚度达到了 $12.4 \mathrm{~nm}$ 。这是由于模板剂的 PMMA 端含有酯基, 导 致酚醛树脂能够同时和 PEO 与 PMMA 端发生相互 作用, 从而形成较厚的墙壁。

为了能够进一步调节有序介观结构、孔径与孔 壁厚度，该课题组 ${ }^{[46]}$ 还合成了有梯度亲水性的 $\mathrm{ABC}$ 两亲性三嵌段共聚物聚环氧乙烷-block-聚甲基

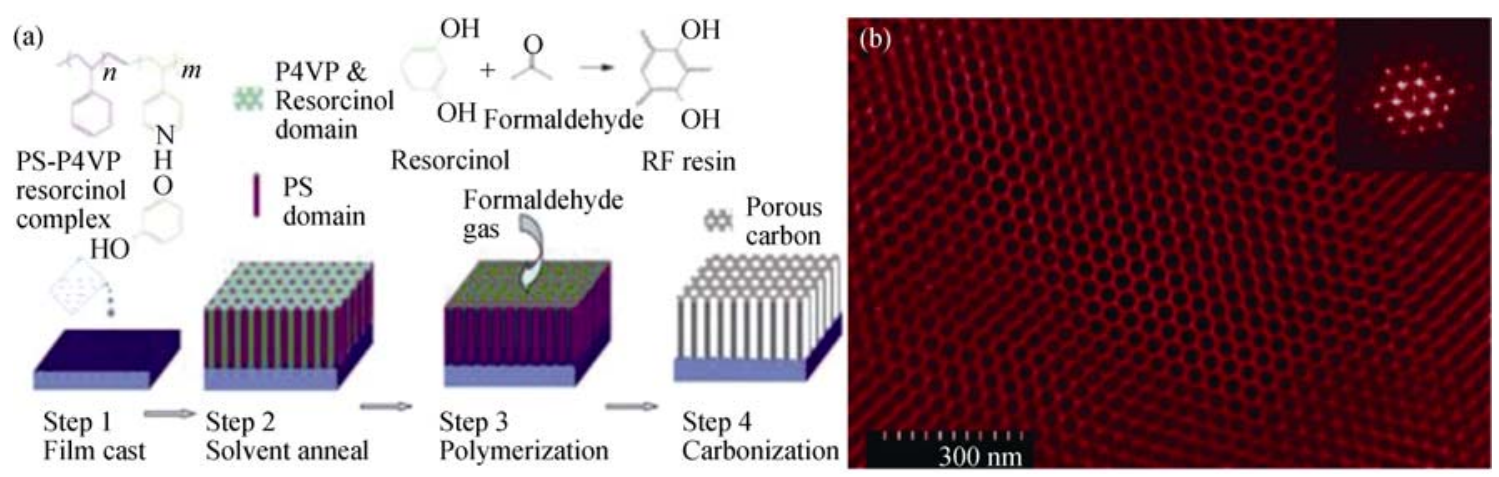

图 3 以 PS- $b$-P4VP 为模板合成有序介孔碳材料的机理示意图(a)和扫描电镜照片(b ${ }^{[43]}$

Fig. 3 Schematic illustration of the formation process of mesoporous carbon by using amphiphilic PS- $b$-P4VP copolymers as template (a) and its corresponding SEM image (b) ${ }^{[43]}$ 
丙烯酸甲酯-block-聚苯乙烯(PEO- $b$-PMMA- $b$-PS)做 模板剂, 低阶酚醛树脂为碳前驱体, 利用 EISA 法合 成了孔径达到 $20 \mathrm{~nm}$ 的面心立方结构的介孔碳材料 (图 4), 墙壁厚度可以在 10 20 nm 范围内进行调控。 通过简单改变前驱体/模板剂比例就可以在如此大 范围内调节墙壁厚度, 这是因为梯度亲水性的 $\mathrm{ABC}$ 三嵌段共聚物模板剂有利于碳前驱体沿着模板剂与 不同亲水链段发生作用。此外, 该课题组 ${ }^{[47]}$ 还改变 了模板剂 PEO- $b$-PMMA 的分子量, 以及其与碳前 驱体的投料比, 通过 EISA 法分别合成了具有二维 六方和面心立方介观结构的介孔碳材料。

\section{3 大孔径介孔金属氧化物材料的合成}

\section{1 介孔氧化钛}

Smarsly 等 ${ }^{[48]}$ 和 Brezesinski 等 ${ }^{[49]}$ 选用嵌段共聚 物聚(乙烯-co-异丁烯)- $b$-聚(环氧乙烷)(简称 KLE) 为 模板剂, $\mathrm{TiCl}_{4}$ 为钛前驱体, 自组装合成了具有体心立 方结构的晶化介孔二氧化钛薄膜, 孔径达到 $14 \mathrm{~nm}$, 晶相为锐钛矿, 孔径大于以往报道的晶化有序介孔 氧化钛材料, 且具有很高的热稳定性。FattakhovaRohlfing 等 ${ }^{[50]}$ 分别使用 KLE 和聚醚类嵌段共聚物 P123 为模板剂, 制备得到的介孔氧化钛薄膜, 发 现两种氧化钛材料具有完全不同的性质。当使用 $\mathrm{KLE}$ 为模板剂, 得到的介孔氧化钛薄膜在低于 $450^{\circ} \mathrm{C}$ 焙烧后具有无定形的骨架, 但经过 $550 \sim 700^{\circ} \mathrm{C}$ 热处 理后可以得到骨架完全晶化的材料。当使用 $\mathrm{P} 123$ 为模板剂, 制备的介孔氧化钛薄膜经过 $400^{\circ} \mathrm{C}$ 焙烧

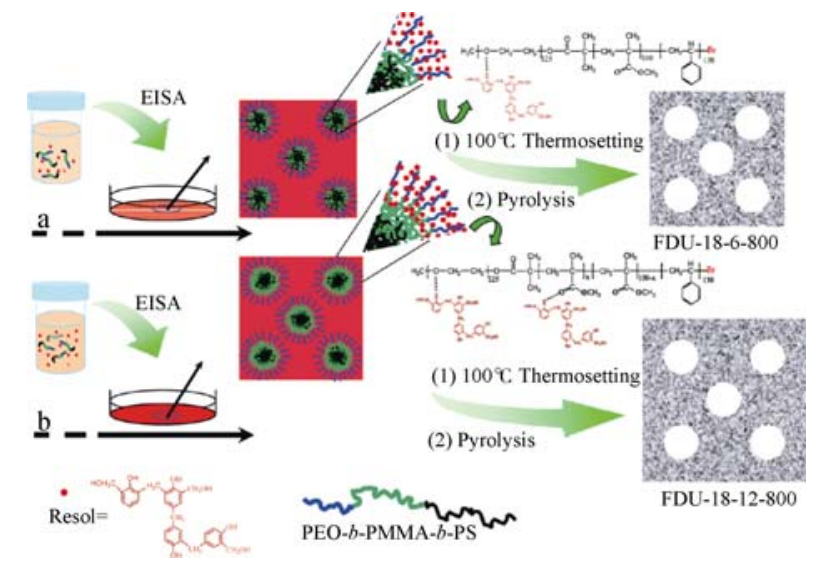

图 4 PEO- $b$-PMMA- $b$-PS 为模板剂合成孔径与墙壁厚度可调 的有序介孔碳材料示意图 ${ }^{[46]}$

Fig. 4 Schematic illustration of the formation process of mesoporous carbon with tunable pore size and wall thickness by using amphiphilic PEO- $b$-PMMA- $b$-PS copolymers as template $^{[46]}$
后, 大约骨架中的一半氧化钛能够晶化, 但是如果 继续提高焙烧温度, 有序介观结构会完全坍塌。这 是由于模板剂KLE与 P123 相比, 疏水性更强, 展现 出更强的自组装驱动力，在自组装过程的初期就会 形成胶束, 大量的氧化钛能够在胶束表面聚集成核, 因此自组装过程较为平缓。在 $450^{\circ} \mathrm{C}$ 焙烧时, 氧化钛 物种仍然呈高度分散态, 故较难结晶, 需要更高的 温度才能晶化。相比之下, P123 的自组装能力较弱, 形成的胶束小而且不稳定，此时氧化钛的异相成核 和均相成核都会发生, 异相成核的部分在 $400^{\circ} \mathrm{C}$ 焙 烧条件下会发生结晶，这样会导致氧化钛骨架具有 晶化和无定形两种状态，继续提高热处理温度就会 引起有序介观结构的圾塌(图 5)。

为了得到性能更为优越的晶化介孔氧化钛材料, Wiesner 等 ${ }^{[51]}$ 开发了软模板和硬模板相结合的方法 (combined assembly by soft and hard chemistries, $\mathrm{CASH}$ ), 他们使用实验室自合成的大分子量两亲性 嵌段共聚物聚环氧乙烷-block-聚异戊二烯(PEO- $b$ PI) 为模板剂, 异丙醇钛为钛前驱体。首先通过 EISA 法得到具有有序介观结构的高分子-无机复合材料, 再在惰性气氛中焙烧, 由于模板剂的疏水端含有 $\mathrm{sp}^{2}$ 型杂化碳, 含碳量也很高, 所以 PI 端在惰性气氛 焙烧后就被碳化成无定型碳，这种原位生成的刚性 碳骨架可以在氧化钛组分高温晶化过程中起到支撑 作用, 避免有序介观结构的坍塌, 待骨架晶化过程 结束后, 在空气气氛下焙烧除去所有的碳组分, 就 可以得到兼具有大孔径与晶化墙壁的介孔氧化钛材 料(图 6)。
$\mathrm{TiO}_{2} /$ Pluronic

$25^{\circ} \mathrm{C}$

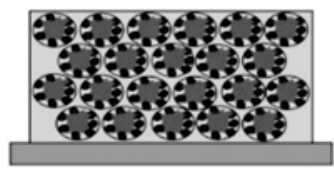

1

$500^{\circ} \mathrm{C}$

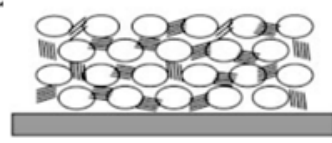

I

$600^{\circ} \mathrm{C}$

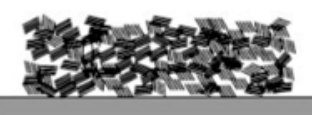

$\mathrm{TiO}_{2} / \mathrm{KLE}$

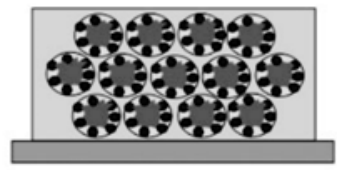

I

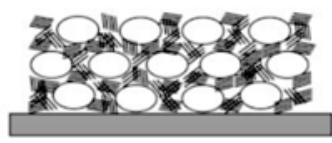

是

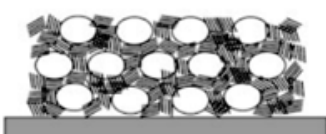

图 5 选用 KLE 或 P123 为模板剂得到介孔二氧化钛材料的 晶化过程示意图 ${ }^{[50]}$

Fig. 5 Schematic illustration of the crystallization process of mesoporous titania by using KLE or P123 as templates ${ }^{[50]}$ 


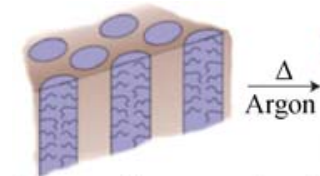
polymer-metal oxide hybrid
Hexagonally arranged
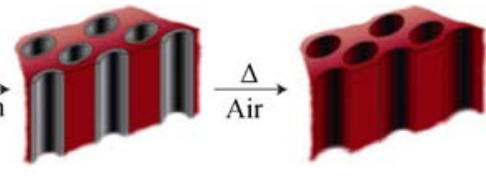

Highly crystalline composite
图 6 利用软一硬模板共组装(CASH)的方法合成高度晶化的 介孔二氧化钛示意图 ${ }^{[51]}$

Fig. 6 Schematic illustration of the formation process of mesoporous titania with high crystallinity via CASH ap$\operatorname{proach}^{[51]}$

Deng 等 ${ }^{[52]}$ 开发了一种配体辅助溶剂挥发诱导 自组装法, 再结合前述的 CASH 后处理法, 合成了 高度晶化的立方相有序介孔氧化钛材料(图 7)。他们 选用 PEO- $b$-PS 为模板剂, 异丙醇钛为钛源, 并在体 系中引入乙酰丙酮, 它可以与异丙醇钛发生螯合作 用, 形成稳定的钛络合物, 从而大大缓解钛前驱体 的快速水解, 促使前驱体和模板剂在溶剂挥发过程 中有足够的时间进行自组装。该方法为晶化介孔金 属氧化物的合成提供了新的思路。

\section{2 介孔氧化铝、氧化铌和氧化铇}

Sanchez 等 ${ }^{[53]}$ 选用大分子量的 KLE22 $\left(\mathrm{BH}_{79-} b\right.$ $\left.\mathrm{EO}_{89}\right)$ 或 $\mathrm{KLE} 23\left(\mathrm{BH}_{320-} b-\mathrm{EO}_{568}\right)$ 为模板剂, 六水合氯 化铝为前驱体，通过提拉法制备了具有面心立方有 序介观结构的 $\gamma-\mathrm{Al}_{2} \mathrm{O}_{3}$ 纳米晶薄膜, $900^{\circ} \mathrm{C}$ 热处理后 还能够保持有序结构不受到破坏, 介孔尺寸达到 $24 \mathrm{~nm}$, 薄膜厚度最大可以调节至 $1 \mu \mathrm{m}$ 。这种兼具 有高度晶化骨架、高度有序介观结构和大孔径尺寸 介孔材料的成功合成同样得益于软模板剂 KLE 较

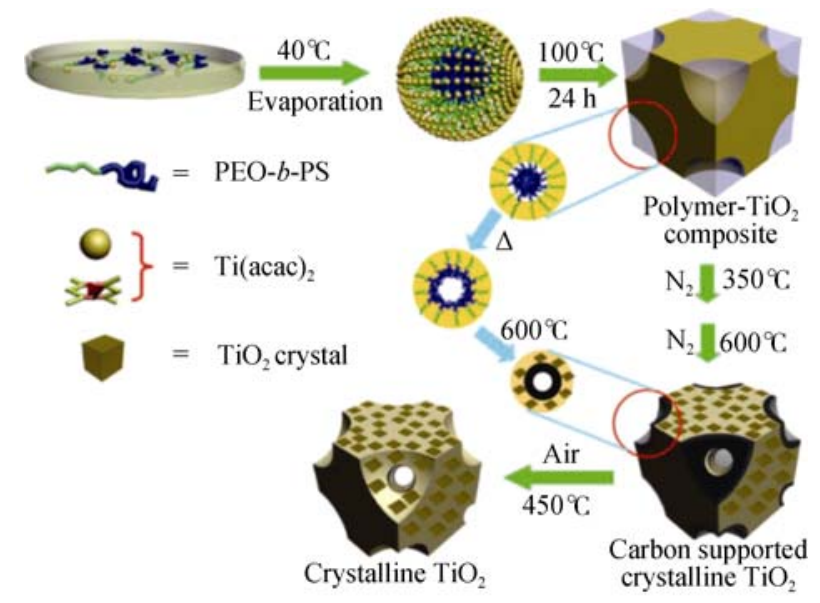

图 7 利用嵌段共聚物 PEO- $b$-PS 为模板剂合成具有简单立方 介观结构的大孔径有序介孔氧化钛机理图 ${ }^{[52]}$

Fig. 7 Schematic illustration of the formation process of mesoporous titania with large pores and simple cubic structure by using amphiphilic PEO- $b$-PS copolymers as template ${ }^{[52]}$
高的含碳量, 可以在高温焙烧晶化过程中给氧化铝 骨架提供有力的支撑, 避免有序介观结构的坍塌, 故展现出很好的热稳定性。

Luo 等 ${ }^{[18]}$ 首次通过酚醛树脂辅助溶剂挥发诱 导自组装(RA-EISA)法合成了具有二维六方结构、 晶化骨架和较大孔径的介孔氧化铌微球(图 8), 这 是首次在 EISA 过程中得到球形形貌的材料。使用 实验室自合成的大分子量 $\mathrm{PEO}_{117}-b-\mathrm{PS}_{221}$ 为模板剂, $\mathrm{NbCl}_{5}$ 为前驱体, 四氢呋喃(THF) 为溶剂, 再添加 少量硝酸。在合成过程中, 在 $\mathrm{THF} /$ 硝酸混合溶液 中形成 PEO- $b$-PS/酚醛树脂/ $\mathrm{NbCl}_{5}$ 复合胶束，随着 THF 的挥发, 胶束的溶解性逐渐降低, 并在 THF 和水的界面力作用下开始缠绕、聚集，首先形成不 规则形状的颗粒，随后转变为规则球形形貌。接下 来, 经过惰性气氛下焙烧碳化高分子组分, 晶化氧 化铌墙壁, 在空气中焙烧除去所有碳组分, 就可以 得到大孔径有序介孔氧化铌微球材料。值得注意的 是, 在晶化过程中, 碳化的 PS 与 resol 组分同时对 有序介观结构产生支撑作用, 起到了较好的效果。 此外, 介孔氧化铌材料具有两种孔径分布(11.4 nm 和 $3.5 \mathrm{~nm}$ ), 分别来源于 PEO- $b$-PS 的模板作用与碳 物种从复合材料中分解所造的孔。合成中低阶酚醛 树脂和 $\mathrm{NbCl}_{5}$ 的比例对最终能否形成氧化铌介孔 微球至关重要。当低阶酚醛树脂和 $\mathrm{NbCl}_{5}$ 的比例过 大时，能通过自组装形成球状结构的有序介孔高 分子(PF)- $\mathrm{Nb}$ 复合物, 但是通过空气焙烧除去碳物 种后, 介观结构很容易坍塌, 这是因为骨架中氧化 铌量太少，不能形成连续的骨架结构来支撑住有 序介观结构。但是当低阶酚醛树脂和 $\mathrm{NbCl}_{5}$ 的比例 太小, 则不能得到有序介孔材料, 这是由于缺乏足 够的酚醛树脂辅助作用, 铌物种和 PEO- $b$-PS 的相 互作用变得较弱, 所以不能够通过自组装作用形 成有序介观结构。合成得到的氧化铌微球材料可应 用于电化学生物传感领域, 对于过氧化氢有着高 灵敏度和快的反应时间。

Brezesinski 等 ${ }^{[54]}$ 选用 KLE 为模板, 氯化铇为前 驱体, 用 EISA 法合成了具有单斜晶系晶体结构的 有序介孔氧化铇薄膜, 孔径尺寸约为 $6 \mathrm{~nm}$, 比表面 积可达 $180 \mathrm{~m}^{2} / \mathrm{g}$, 墙壁由尺寸为 $12 \sim 14 \mathrm{~nm}$ 的晶粒组 成。 $\mathrm{Li}$ 等 ${ }^{[55]}$ 同样使用氯化铇为前驱体, 但选择的模 板剂为 PEO- $b$-PS, 他们开发了一种简单的模板碳化 辅助晶化的方法, 利用低温碳化使得疏水端 PS 链 段原位转化成的无定形碳, 来作为氧化铇墙壁的刚 性支撑，制备了具有面心立方结构的有序介孔氧化 铇材料(图 9), 孔径尺寸可以达到 $10.9 \mathrm{~nm}$, 比表面 


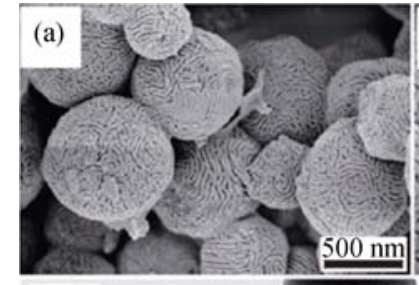

(b)

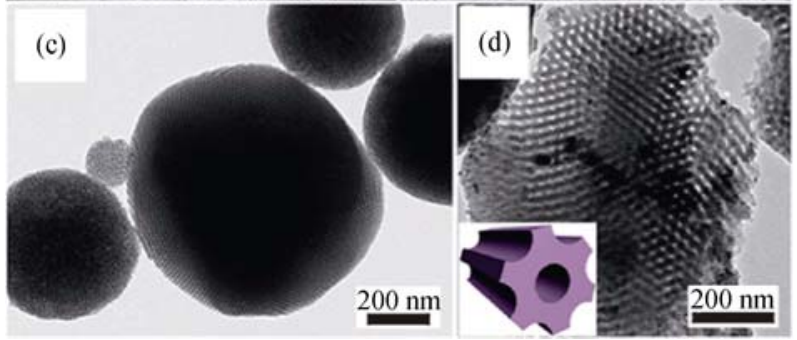

图 8 以 PEO- $b$-PS 为模板剂通过 RA-EISA 法合成介孔氧化 铌微球的场发射扫描电镜照片 $(\mathrm{a}, \mathrm{b})$ 和透射电镜照片 $(\mathrm{c}, \mathrm{d})^{[18]}$ Fig. 8 SEM (a,b) and TEM (c,d) images of mesoporous niobia spheres via RA-EISA approach by using amphiphilic PEO- $b$-PS copolymers as template ${ }^{[18]}$
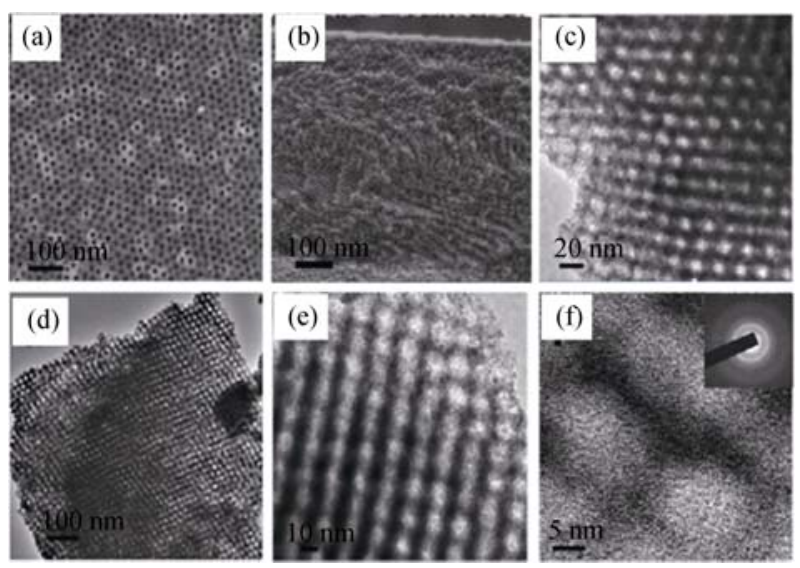

图 9 介孔氧化铇材料的场发射扫描电镜照片 $(a, b)$ 和透射电 镜照片(c f f $)^{[55]}$

Fig. 9 SEM (a,b) and TEM (c-f) images of mesoporous $\mathrm{WO}_{3}{ }^{[55]}$

积约为 $121 \mathrm{~m}^{2} / \mathrm{g}$, 结晶度较高, 呈单斜晶相, 这种 材料具有非常优异的 $\mathrm{H}_{2} \mathrm{~S}$ 气体气敏特性, 对 $50 \times$ $10^{-6}$ 的气体响应和恢复时间仅有 $2 \mathrm{~s}$ 和 $38 \mathrm{~s}$ 。

\section{3 介孔氧化锡、氧化铈、氧化锆和氧化铱}

$\mathrm{Kim}$ 等 ${ }^{[56]}$ 选用两亲性接枝共聚物聚氯乙烯graft-聚氧乙烯丙烯酸甲酯(PVC- $g$-POEM) 为模板剂, 二氯化锡为前驱体, 使用旋涂法, 经过 EISA 过程, 可以在 FTO 基底上得到金红石晶相的介孔氧化锡 薄膜, 平均晶粒尺寸约为 $12.4 \mathrm{~nm}$, 孔径尺寸高达 $30 \sim 40 \mathrm{~nm}$, 薄膜厚度为 $400 \mathrm{~nm}$ 。该薄膜材料可应用 于染料敏化太阳能电池领域, 展现出了较好的性能。

Brezesinski 等在介孔氧化铈的合成方面也做了 很多工作, 他们选择 $\mathrm{KLE}$ 为模板剂, $\mathrm{CeCl}_{3} \cdot 7 \mathrm{H}_{2} \mathrm{O}$ 为
铈前驱体，通过提拉法合成了具有晶化骨架以及三 维立方相有序介观结构的介孔氧化铈薄膜, 孔径尺 寸高达 16 18 $\mathrm{nm}^{[57]}$ 。该材料的墙壁厚度也达到了 10 15 nm, 在高温晶化过程中可以提供更刚性的骨 架, 经过 $800^{\circ} \mathrm{C}$ 热处理后发现材料还保持着较好的 介观有序性，此时墙壁由 12 13 nm 的晶粒组成。通 过计算, 其比表面积约为 $121 \mathrm{~m}^{2} / \mathrm{g}$ 。

KLE 同样可以作模板来合成大孔径氧化锆材料, Antonietti 等 ${ }^{[58}$ 选用氯化锆为前驱体, 采用提拉法在 一定的相对湿度 $(10 \%$ 20\%)下合成了具有高度晶化孔 壁的无序介孔氧化锆薄膜, 墙壁由直径 $10 \mathrm{~nm}$ 左右的 氧化锆晶粒构成。随后, Fang 等 ${ }^{[59]}$ 改善了大孔径介 孔氧化锆材料的介观有序性, 他们选用大分子量两 亲性嵌段共聚物聚环氧乙烷- $b$-聚丁二烯(PEO- $b$-PB) 为模板剂, 氯化锆作锆源, 同样通过提拉法合成了具 有二维六方介观结构及晶化骨架的有序介孔氧化锆 薄膜, 孔径达到 $7.1 \mathrm{~nm}$, 比表面积约为 $151 \mathrm{~m}^{2} / \mathrm{g}$ 。

Kraehnert 等 ${ }^{[60]}$ 选用大分子量 PEO-PB-PEO 作 为模板剂, 醋酸铱为前驱体, 通过提拉法合成具有 有序介观结构氧化铱一高分子复合材料。再经过 $300^{\circ} \mathrm{C}$ 焙烧除去模板剂后, 可以得到较大孔径 $(16 \mathrm{~nm})$ 的有 序介孔氧化铱薄膜, 比表面积可达 $140 \mathrm{~m}^{2} / \mathrm{g}$ 。他们 还研究了焙烧温度对于薄膜性质的影响, 发现随着 焙烧温度的升高, 比表面积和薄膜厚度都显著地降 低, 而晶粒尺寸却不断增大。此外, 研究表明 $450^{\circ} \mathrm{C}$ 焙烧处理后的氧化铱薄膜材料对于析氧反应 (oxygen evolution reaction) 展现出较好的电催化活 性及稳定性。

\section{4 大孔径介孔金属材料的合成}

金属铂是一种石油化工中常用的催化剂, 制备 介孔结构的铂材料可以有效提高其催化效率。 Yamauchi 等 ${ }^{[61]}$ 选用大分子量 PEO-b-PS 作为模板剂, 氯铂酸为前驱体, 使用电化学沉积法将铂纳米粒子 排列成三维互通的笼状孔结构。该材料的孔径达到 $15 \mathrm{~nm}$ 。随后，该课题组 ${ }^{[62]}$ 使用同样的方法在金基底 上制备得到高度有序的铂薄膜材料, 通过改变模板 剂和铂前驱体的比例, 可以得到三维笼型、二维六 方或层状结构的介观结构, 这种材料展现出较好的 电催化活性。

Watkins 等 ${ }^{[63]}$ 选用 PEO- $b$-PS 为模板剂, 引导功 能化的金纳米粒子进行自组装, 可以得到立方、二 维六方和层状等有序金-高分子复合介观结构, 其 中金的质量分数可以达到 $30 \%$ 。但是，该有序结构 
不能脱离模板剂组分独立存在。

Ho 等 ${ }^{[64]}$ 选用聚苯乙烯-block-聚乙醇酸(PS- $b$ PLLA)为模板剂, 首先使其形成单相的有序立方双 连续介观结构, 再通过水解处理, 除去 PLLA 组分, 得到有序排列的孔道。接下来使用电沉积法在孔道 里面填充镍前驱体, 最后通过四氢呋喃萃取除去 PS 组分后就可以得到具有立方双连续介观结构的有序 介孔镍材料, 其比表面积约为 $25 \mathrm{~m}^{2} / \mathrm{g}$ 。

\section{5 总结与展望}

介孔材料经过二十多年的研究与发展, 其合成 方法、制备机理以及孔性质的调控等已经日趋成熟, 相继报道了具有各种骨架组成、孔道结构以及功能 的介孔材料。其发展方向也逐渐转向介孔材料的实 际应用, 而目前介孔材料的孔径相对较小, 不利于 大体积客体分子的进出与传递, 并且难以制备具有 优良物理化学性能的晶化介孔材料。这两点是介孔 材料能否得到进一步应用的瓶颈, 而设计和合成新 型两亲性嵌段共聚物作为合成介孔材料的软模板剂, 能够避免传统商业化模板剂的缺陷, 可以得到具有 较大孔径和晶化墙壁的有序介孔材料, 进一步拓宽 介孔材料的应用范围。

从理论上说, 介孔材料的孔径可以通过对模板 剂疏水端分子量大小的控制来进行任意调控, 而改 变模板剂的亲水/疏水体积比, 又可以得到迥然不同 的有序介观结构。所以, 模板剂的设计与制备尤为 关键, 需要广大科研工作者从高分子化学的角度出 发, 设计各种分子量大小、亲/疏水基才等不同的两 亲性嵌段共聚物, 并通过不同的聚合方法制备出 来。并以得到的嵌段共聚物作为模板剂, 根据实际 应用的具体需要, 引导合成孔径和墙壁厚度可调、 热稳定性好、骨架高度晶化的介孔材料, 从而进一 步推动介孔材料在各个领域的应用。

\section{参考文献:}

[1] SHI Y, WAN Y, ZHAO D. Ordered mesoporous non-oxide materials. Chem. Soc. Rev., 2011, 40(7): 3854-3878.

[2] ZHAO D, FENG J, HUO Q, et al. Triblock copolymer syntheses of mesoporous silica with periodic 50 to 300 angstrom pores. Science, 1998, 279(5350): 548-552.

[3] BECK J S, VARTULi J C, ROTH W J, et al. A new family of mesoporous molecular sieves prepared with liquid crystal templates. J. Am. Chem. Soc., 1992, 114(27): 10834-10843.

[4] MENG Y, GU D, ZHANG F, et al. A Family of highly ordered mesoporous polymer resin and carbon structures from organic-organic self-sssembly. Chem. Mater, 2006, 18(18): $4447-4464$.

[5] ZHAI Y, DOU Y, ZHAO D, et al. Carbon materials for chemical capacitive energy storage. Adv. Mater., 2011, 23(42): 4828-4850.

[6] LIU Y, LAN K, BAGABAS A A, et al. Ordered macro/mesoporous $\mathrm{TiO} 2$ hollow microspheres with highly crystalline thin shells for high-efficiency photoconversion. Small, 2016, 12(7): 860-867.

[7] WANG C, LI X, XI X, et al. Bimodal highly ordered mesostructure carbon with high activity for $\mathrm{Br}_{2} / \mathrm{Br}^{-}$redox couple in bromine based batteries. Nano Energy, 2016, 21: 217-227.

[8] LIU H, LI W, SHEN D, et al. Graphitic carbon conformal coating of mesoporous $\mathrm{TiO}_{2}$ hollow spheres for high-performance lithium ion battery anodes. J. Am. Chem. Soc., 2015, 137(40): 13161-13166.

[9] ZHOU W, GAO H,GOODENOUGH J B. Low-cost hollow mesoporous polymer spheres and all-solid-state lithium, sodium batteries. Adv. Energy Mater, 2016, 6(1):1501802.

[10] DENG Y, CAI Y, SUN Z, et al. Multifunctional mesoporous composite microspheres with well-designed nanostructure: a highly integrated catalyst system. J. Am. Chem. Soc., 2010, 132(24): 8466-8473.

[11] GARG S, SONI K, AJEETH PRABHU T, et al. Effect of ordered mesoporous Zr SBA-15 support on catalytic functionalities of hydrotreating catalysts 2 . Variation of molybdenum and promoter loadings. Catal. Today, 2016, 261: 128-136.

[12] DUTTA B, BISWAS S, SHARMA V, et al. Mesoporous manganese oxide catalyzed aerobic oxidative coupling of anilines to aromatic azo compounds. Angew. Chem. Int. Ed., 2016, 55(6): 2171-2175.

[13] JOO S H, PARK J Y, TSUNG C K, et al. Thermally stable $\mathrm{Pt} / \mathrm{mesoporous} \mathrm{silica} \mathrm{core-shell} \mathrm{nanocatalysts} \mathrm{for} \mathrm{high-temperature}$ reactions. Nat. Mater., 2009, 8(2): 126-131.

[14] EGODAWATTE S, DATT A, BURNS E A, et al. Chemical insight into the adsorption of chromium(III) on iron oxide/mesoporous silica nanocomposites. Langmuir, 2015, 31(27): 7553-7562.

[15] FAN J, YU C, GAO F, et al. Cubic mesoporous silica with large controllable entrance sizes and advanced adsorption properties. Angew. Chem. Int. Ed., 2003, 42(27): 3146-3150.

[16] TENG W, WU Z, FAN J, et al. Amino-functionalized ordered mesoporous carbon for the separation of toxic microcystin-LR. $J$. Mater. Chem., 2015, 3(37): 19168-19176.

[17] WU C, LIANG Y, YANG K, et al. Clickable periodic mesoporous organosilica monolith for highly efficient capillary chromatographic separation. Anal. Chem., 2016, 88(3): 1521-1525.

[18] LUO W, LI Y, DONG J, et al. A resol-assisted co-assembly approach to crystalline mesoporous niobia spheres for electrochemi- 
cal biosensing. Angew. Chem. Int. Ed., 2013, 52(40): 10505-10510.

[19] GE X, SUN L, MA B, et al. Simultaneous realization of $\mathrm{Hg}^{2+}$ sensing, magnetic resonance imaging and upconversion luminescence in vitro and in vivo bioimaging based on hollow mesoporous silica coated UCNPs and ruthenium complex. Nanoscale, 2015, 7(33): 13877-13887.

[20] LI Z, CLEMENS D L, LEE B Y, et al. Mesoporous silica nanoparticles with $\mathrm{pH}$-sensitive nanovalves for delivery of moxifloxacin provide improved treatment of lethal pneumonic tularemia. ACS Nano, 2015, 9(11): 10778-10789.

[21] LIU J, LUO Z, ZHANG J, et al. Hollow mesoporous silica nanoparticles facilitated drug delivery via cascade $\mathrm{pH}$ stimuli in tumor microenvironment for tumor therapy. Biomaterials, 2016, 83: $51-65$.

[22] WANG Y, GU H. Core shell-type magnetic mesoporous silica nanocomposites for bioimaging and therapeutic agent delivery. $A d v$. Mater, 2015, 27(3): 576-585.

[23] SCHÜTH F. Non-siliceous mesostructured and mesoporous materials. Chem. Mater., 2001, 13(10): 3184-3195.

[24] WIDENMEYER M,ANWANDER R. Pore size control of highly ordered mesoporous silica MCM-48. Chem. Mater, 2002, 14(4): $1827-1831$.

[25] YU C, FAN J, TIAN B, et al. Synthesis of mesoporous silica from commercial poly(ethylene oxide)/poly(butylene oxide) copolymers: toward the rational design of ordered mesoporous materials. The Journal of Physical Chemistry B, 2003, 107(48): 13368-13375.

[26] HUO Q, MARGOLESE D I,STUCKY G D. Surfactant control of phases in the synthesis of mesoporous silica-based materials. Chem. Mater., 1996, 8(5): 1147-1160.

[27] KRESGE C T, LEONOWICZ M E, ROTH W J, et al. Ordered mesoporous molecular sieves synthesized by a liquid-crystal template mechanism. Nature, 1992, 359(6397): 710-712.

[28] GAO C, SAKAMOTO Y, SAKAMOTO K, et al. Synthesis and characterization of mesoporous silica AMS-10 with bicontinuous cubic Pn3m symmetry. Angew. Chem. Int. Ed., 2006, 45(26): 4295-4298.

[29] CHE S, GARCIA-BENNETT A E, YOKOI T, et al. A novel anionic surfactant templating route for synthesizing mesoporous silica with unique structure. Nat. Mater, 2003, 2(12): 801-805.

[30] GARCIA-BENNETT A E, KUPFERSCHMIDT N, SAKAMOTO $\mathrm{Y}$, et al. Synthesis of mesocage structures by kinetic control of self-assembly in anionic surfactants. Angew. Chem. Int. Ed., 2005, 44(33): 5317-5322.

[31] ZHAO D, HUO Q, FENG J, et al. Nonionic triblock and star diblock copolymer and oligomeric surfactant syntheses of highly ordered, hydrothermally stable, mesoporous silica structures. J. Am.
Chem. Soc., 1998, 120(24): 6024-6036.

[32] FAN J, YU C, LEI J, et al. Low-temperature strategy to synthesize highly ordered mesoporous silicas with very large pores. $\mathrm{J}$. Am. Chem. Soc., 2005, 127(31): 10794-10795.

[33] MA G, YAN X, LI Y, et al. Ordered nanoporous silica with periodic 30-60 nm pores as an effective support for gold nanoparticle catalysts with enhanced lifetime. J. Am. Chem. Soc., 2010, 132(28): 9596-9597.

[34] BRINKER C J, LU Y, SELLINGER A, et al. Evaporation-induced self-assembly: nanostructures made easy. Adv. Mater., 1999, 11(7): 579-585.

[35] DENG Y, CAI Y, SUN Z, et al. Controlled synthesis and functionalization of ordered large-pore mesoporous carbons. Adv. Funct. Mater, 2010, 20(21): 3658-3665.

[36] WEI J, WANG H, DENG Y, et al. Solvent evaporation induced aggregating assembly approach to three-dimensional ordered mesoporous silica with ultralarge accessible mesopores. J. Am. Chem. Soc., 2011, 133(50): 20369-20377.

[37] WEI J, YUE Q, SUN Z, et al. Synthesis of dual-mesoporous silica using non-ionic diblock copolymer and cationic surfactant as co-templates. Angew. Chem. Int. Ed., 2012, 51(25): 6149-6153.

[38] WANG C, WEI J, YUE Q, et al. A shear stress regulated assembly route to silica nanotubes and their closely packed hollow mesostructures. Angew. Chem. Int. Ed., 2013, 52(44): 11603-11606.

[39] YU K, SMARSLY B, BRINKER C J. Self-Assembly and characterization of mesostructured silica films with a 3D arrangement of isolated spherical mesopores. Adv. Funct. Mater, 2003, 13(1): $47-52$.

[40] SMARSLY B, XOMERITAKIS G, YU K, et al. Microstructural characterization of polystyrene-block-poly(ethylene oxide)-templated silica films with cubic-ordered spherical mesopores. Langmuir, 2003, 19(18): 7295-7301.

[41] YU K, HURD A J, EISENBERG A, et al. Syntheses of silica/ polystyrene-block-poly (ethylene oxide) films with regular and reverse mesostructures of large characteristic length scales by solvent evaporation-induced self-assembly. Langmuir, 2001, 17(26): 7961-7965.

[42] DENG Y, YU T, WAN Y, et al. Ordered mesoporous silicas and carbons with large accessible pores templated from amphiphilic diblock copolymer poly(ethylene oxide)-b-polystyrene. J. Am. Chem. Soc., 2007, 129(6): 1690-1697.

[43] LIANG C, HONG K, GUIOCHON G A, et al. Synthesis of a large-scale highly ordered porous carbon film by self-assembly of block copolymers. Angew. Chem. Int. Ed., 2004, 43(43): 5785-5789.

[44] RODRIGUEZ A T, LI X, WANG J, et al. Facile synthesis of nanostructured carbon through self-assembly between block copolymers and carbohydrates. Adv. Funct. Mater, 2007, 17(15): 
2710-2716.

[45] DENG Y, LIU C, GU D, et al. Thick wall mesoporous carbons with a large pore structure templated from a weakly hydrophobic PEO-PMMA diblock copolymer. J. Mater. Chem., 2008, 18(1): 91-97.

[46] ZHANG J, DENG Y, WEI J, et al. Design of amphiphilic ABC triblock copolymer for templating synthesis of large-pore ordered mesoporous carbons with tunable pore wall thickness. Chem. Mater., 2009, 21(17): 3996-4005.

[47] WEI J, DENG Y, ZHANG J, et al. Large-pore ordered mesoporous carbons with tunable structures and pore sizes templated from poly(ethylene oxide)-b-poly(methyl methacrylate). Solid State Sci., 2011, 13(4): 784-792.

[48] SMARSLY B, GROSSO D, BREZESINSKI T, et al. Highly crystalline cubic mesoporous $\mathrm{TiO}_{2}$ with $10-\mathrm{nm}$ pore diameter made with a new block copolymer template. Chem. Mater, 2004, 16(15): 2948-2952.

[49] BREZESINSKI T, GROENEWOLT M, GIBAUD A, et al. Evaporation-induced self-assembly (EISA) at its limit: ultrathin, crystalline patterns by templating of micellar monolayers. Adv. Mater., 2006, 18(17): 2260-2263.

[50] FATTAKHOVA-ROHLFING D, WARK M, BREZESINSKI T, et al. Highly organized Mesoporous $\mathrm{TiO}_{2}$ films with controlled crystallinity: A Li-insertion study. Adv. Funct. Mater, 2007, 17(1): 123-132.

[51] LEE J, CHRISTOPHER ORILALL M, WARREN S C, et al. Direct access to thermally stable and highly crystalline mesoporous transition-metal oxides with uniform pores. Nat. Mater, 2008, 7(3): 222-228.

[52] ZHANG J, DENG Y, GU D, et al. Ligand-assisted assemblyapproach to synthesize large-pore ordered mesoporous titania with thermally stable and crystalline Framework. Adv. Energy Mater, 2011, 1(2): 241-248.

[53] KUEMMEL M, GROSSO D, BOISSIRE C, et al. Thermally stable nanocrystalline $\gamma$-alumina layers with highly ordered 3D mesoporosity. Angew. Chem. Int. Ed., 2005, 44(29): 4589-4592.

[54] BREZESINSKI T, FATTAKHOVA ROHLFING D, SALLARD S, et al. Highly crystalline $\mathrm{WO}_{3}$ thin films with ordered 3D mesoporosity and improved electrochromic performance. Small, 2006,
2(10): 1203-1211.

[55] LI Y, LUO W, QIN N, et al. Highly ordered mesoporous tungsten oxides with a large pore size and crystalline framework for $\mathrm{H}_{2} \mathrm{~S}$ sensing. Angew. Chem. Int. Ed., 2014, 53(34): 9035-9040.

[56] PARK T, AHN S, ROH D, et al. Multifunctional organized mesoporous tin oxide films templated by graft copolymers for dye-Sensitized solar cells. ChemSusChem, 2014, 7(7): 2037-2047.

[57] BREZESINSKI T, WANG J, SENTER R, et al. On the correlation between mechanical flexibility, nanoscale structure, and charge storage in periodic mesoporous $\mathrm{CeO}_{2}$ thin films. ACS Nano, 2010, 4(2): 967-977.

[58] BREZESINSKI T, ANTONIETTI M, GROENEWOLT M, et al. The generation of mesostructured crystalline $\mathrm{CeO}_{2}, \mathrm{ZrO}_{2}$ and $\mathrm{CeO}_{2}-\mathrm{ZrO}_{2}$ films using evaporation-induced self-assembly. New $\mathrm{J}$. Chem., 2005, 29(1): 237-242.

[59] FANG H, WAN T, SHI W, et al. Design and synthesis of large-pore p6mm mesoporus zirconia thin films templated by a novel block copolymer. J. Non-Cryst. Solids, 2007, 353(16/17): $1657-1661$.

[60] ORTEL E, REIER T, STRASSER P, et al. Mesoporous $\mathrm{IrO}_{2}$ films templated by PEO-PB-PEO block-copolymers: self-assembly, crystallization behavior, and electrocatalytic performance. Chem. Mater, 2011, 23(13): 3201-3209.

[61] YAMAUCHI Y, SUGIYAMA A, MORIMOTO R, et al. Mesoporous platinum with giant mesocages templated from lyotropic liquid crystals consisting of diblock copolymers. Angew. Chem. Int. Ed., 2008, 47(29): 5371-5373.

[62] TAKAI A, YAMAUCHI Y, KURODA K. Tailored electrochemical synthesis of 2D-hexagonal, lamellar, and cage-type mesostructured Pt thin films with extralarge periodicity. J. Am. Chem. Soc., 2010, 132(1): 208-214.

[63] LIN Y, DAGA V K, ANDERSON E R, et al. Nanoparticle-driven assembly of clock copolymers: a simple route to ordered hybrid materials. J. Am. Chem. Soc., 2011, 133(17): 6513-6516.

[64] HSUEH H Y, HUANG Y C, HO R M, et al. Nanoporous gyroid nickel from block copolymer templates via electroless plating. $A d v$. Mater, 2011, 23(27): 3041-3046. 